\title{
Small Bowel Obstruction Caused by the Ingestion of a Wooden Toothpick: The CT findings and a Literature Review
}

\author{
Junichi Izumi ${ }^{1}$, Kimihiko Satoh ${ }^{2}$, Wataru Iwasaki ${ }^{2}$, Takaya Miura ${ }^{3}$ and Shusei Fujimori ${ }^{3}$
}

\begin{abstract}
We present a case in which the accidental ingestion of a toothpick caused duodenal perforation and small intestinal obstruction. A 58-year-old man visited our emergency room with acute abdominal pain. Computed tomography (CT) showed obstructive ileus as well as a foreign body penetrating the duodenum, which was identified as a toothpick and removed endoscopically. Unenhanced CT was superior in detecting the object. The patient has been doing well since the operation.
\end{abstract}

Key words: toothpick ingestion, small bowel obstruction, duodenal perforation, computed tomography, CT

(Intern Med 56: 657-660, 2017)

(DOI: 10.2169/internalmedicine.56.7463)

\section{Introduction}

Toothpick ingestion is a rare event, but it is a medical emergency because it represents a potentially life-threatening event (1). Unfortunately, the detectability of an ingested toothpick on imaging studies is not sufficient. We herein describe a case of small bowel obstruction caused by an accidentally swallowed toothpick and emphasize the usefulness of unenhanced computed tomography (CT) in visualizing the wooden foreign body.

\section{Case Report}

A 58-year-old man presented to our emergency room with acute abdominal pain. A physical examination revealed mild distention of the abdomen and tenderness without signs of peritonitis. His vital signs were stable, with a blood pressure of 163/95 mmHg and a pulse of 101 beats per minute. His blood work was within the normal limits with the exception of a white blood cell count of $12,100 / \mathrm{mm}^{3}$ and a C-reactive protein level of $0.42 \mathrm{mg} / \mathrm{dL}$. An abdominal X-ray showed small bowel distention, which indicated the possibility of ileus. Abdominal and pelvic computed tomography (CT) was then performed.

Both unenhanced and enhanced CT identified a small bowel obstruction with findings of dilatation and fluid collection in the proximal part of the small intestine (Fig. 1A). A caliber change of the small intestine was found at the right upper abdomen. We regarded the portion as the obstructed or stenotic site of the small bowel. On unenhanced CT, axial images depicted a tiny high density area accompanied by fat stranding near the narrowed intestine (Fig. 1B). At a more cephalad level, the high density structure was found to be located within the duodenum. Reformatted coronal and sagittal images clearly demonstrated that the high density area was a long and straight thin structure, which was located both inside and outside the duodenum (Fig. 1C-1D). Based on these findings, we made a diagnosis of duodenal perforation caused by the accidental swallowing of a fish bone. The density of the soft tissue around the high density object suggested that it was granulation tissue, which had led to adhesion to the intestine and subsequent small bowel obstruction. Interestingly, the detectability of the high density structure was worse on enhanced CT (Fig. 1E). Neither ascites nor abscesses were observed. Incidentally, we found that the patient had a variation of the duodenal route: the proximal 3rd portion of the duodenum

${ }^{1}$ Department of Radiology, Yokote Municipal Hospital, Japan, ${ }^{2}$ Department of Surgery, Yokote Municipal Hospital, Japan and ${ }^{3}$ Department of Internal Medicine, Yokote Municipal Hospital, Japan

Received for publication March 23, 2016; Accepted for publication July 11, 2016

Correspondence to Dr. Junichi Izumi, j_izumi@yokote.mhp.jp 

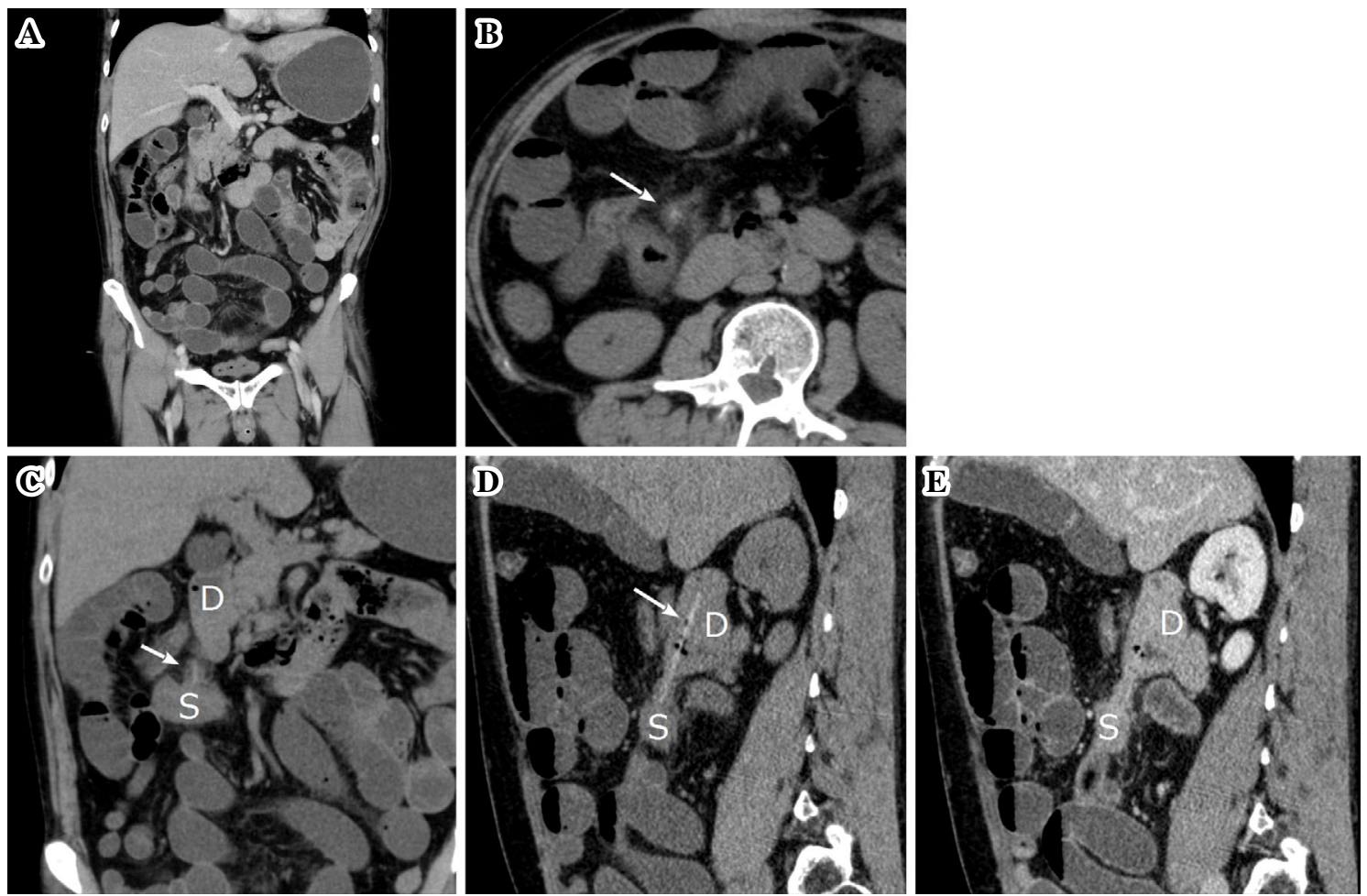

Figure 1. A computed tomography (CT) scan showing the distended and fluid-filled small bowel (A), which is consistent with small bowel obstruction. An unenhanced axial CT image just below the duodenum shows a tiny high density dot surrounded by soft tissue density (B: arrow). Reconstructed coronal (C) and sagittal (D) images afford a better understanding of the linear high density structure (arrow). Our preoperative diagnosis was an accidentally ingested fish bone penetrating the duodenum and causing small bowel obstruction. Interestingly, the foreign body was obscured on enhanced CT due to the influence of inflammatory and normal tissue enhancement (E). $D$ : duodenum, $S$ : small intestine

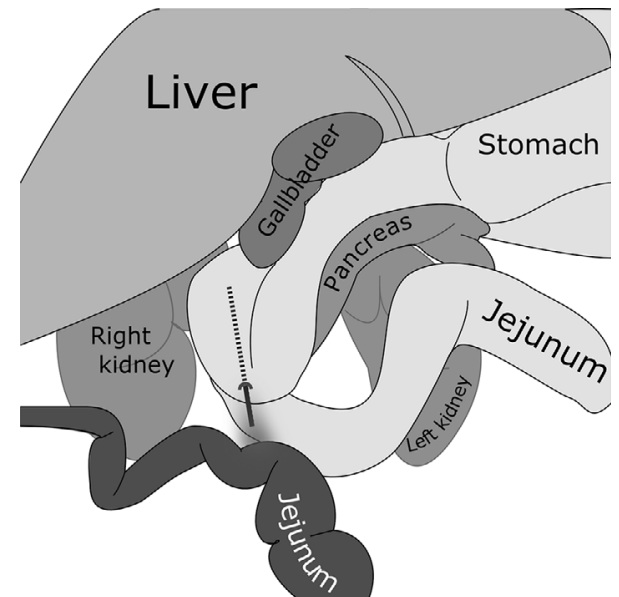

Figure 2. An illustration of the relationship between the foreign object penetrating the duodenum and the small bowel near the duodenum. Note that the patient has an anomalous duodenal route.

went upward behind the 2nd part, and then descended again at the back of the proximal 3 rd portion of the duodenum (Fig. 2).

He was admitted and a nasogastric tube was used to achieve decompression. An endoscopic examination of the upper gastrointestinal tract performed the next day revealed a wooden toothpick, not a fish bone, penetrating the duodenal wall slightly proximally to the papilla of Vater (Fig. 3A). The foreign body, which he could not recall swallowing, was successfully retrieved with an endoscopic snare (Fig. 3B, C). The patient received 9 days of fast care with the administration of an anti-ulcer agent, and was discharged uneventfully after 15 days. A follow-up CT examination that was performed one month later showed no signs of small bowel obstruction and the complete disappearance of the soft tissue density near the duodenum that was seen on the previous CT images.

\section{Discussion}

Toothpick ingestion is uncommon. The U.S. Consumer Product Safety Commission National Injury Clearinghouse from 1979 to 1982 estimated that the incidence of internal organ perforation due to toothpick ingestion was 0.2 per 100,000 persons (2). In most cases, ingested foreign bodies pass through the gastrointestinal tract uneventfully and less than $1 \%$ of cases cause perforation $(3,4)$. When only sharp, pointed objects are considered, the perforation rate increases to $15-35 \%$ (3). According to a recent review, the incidence 

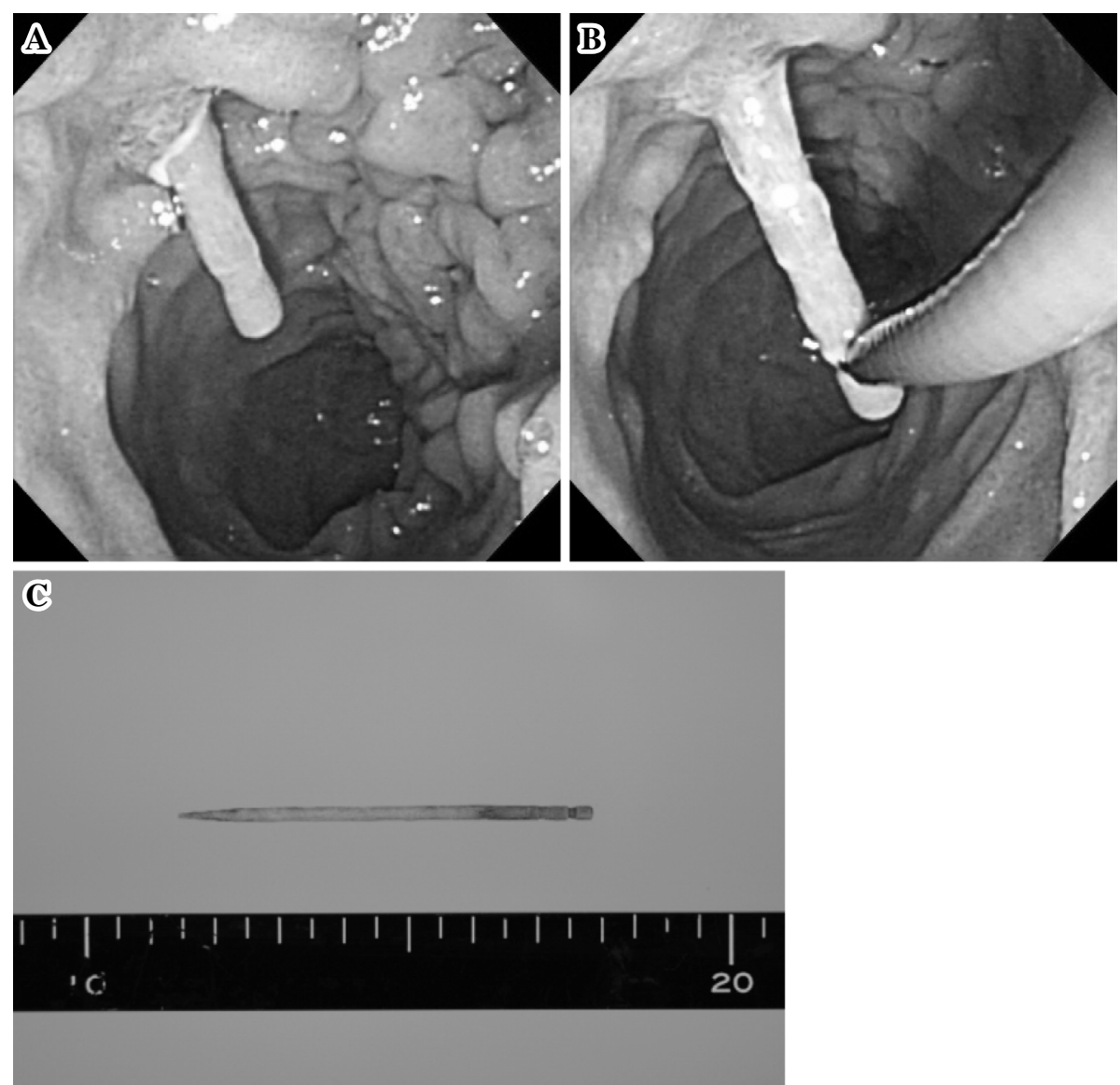

Figure 3. An endoscopic examination performed the day after admission revealed a wooden toothpick penetrating the duodenum (A). The endoscopic removal of the toothpick $(6.5 \mathrm{~cm}$ in length) was accomplished using the snare technique $(\mathrm{B}, \mathrm{C})$.

of toothpick-related gut perforation is as high as $80 \%$ (1). Risk factors for toothpick ingestion include the habitual chewing of toothpicks, rapid eating, mental disorder, the use of dentures, and alcohol consumption $(1,2,5)$. The most common site of toothpick lodgment or perforation is the duodenum $(23 \%)$, followed by the stomach $(20 \%)$, small intestine (18\%), and sigmoid colon (16\%) (1). Toothpick ingestion can cause gastrointestinal bleeding, gut perforation, obstruction, sepsis, abscess (in the liver, peritoneal cavity, or retroperitoneum), or fistula formation $(2,6,7)$. The migration of toothpicks may be found in the pleura, liver, free peritoneal space, retroperitoneal space, pericardium, urinary bladder, aorta, inferior vena cava, coronary artery, pancreas, ureter, hepatoduodenal ligament, vena portae, lungs, and kidneys (1).

Toothpick ingestion is a life-threatening event, with a mortality rate of $9.6-18 \%(1,6)$. Although an early diagnosis and treatment are crucial for reducing morbidity and mortality, a correct diagnosis may be very difficult. One of the reasons for the difficulty is that most patients do not recall swallowing a toothpick. Only $12 \%$ of patients were able to remember swallowing a toothpick, and among these patients, the onset of symptoms occurred from less than one day to 15 years after the event (6). Moreover, the symptoms associated with toothpick ingestion are non-specific, such as abdominal pain, fever, nausea, or diarrhea (1).

In the present case, the toothpick penetrating the duodenum was represented as a linear high density structure on unenhanced CT. A high density is a common feature of wooden foreign bodies on CT (as long as they contain water) (8). In fact, in other reports of accidentally swallowed toothpicks, they were most often observed as high density areas. The discrimination of a water-filled toothpick from a fish bone, which is one of the most common accidentally-swallowed foreign bodies (9), would be difficult because they have a similar appearance on CT (a fish bone penetration appears as a linear high density area surrounded by an area of inflammation) (10). In a similar vein, the potential pitfalls in detecting a toothpick on CT would be the presence of positive bowel contrast, artifacts related to fecal material, and contrast-enhanced small blood vessels (9). Interestingly, in our case, the toothpick was indistinct on the CT images that were taken after intravenous contrast administration. We hypothesize that a good contrast effect in the granulation tissue (which was caused by the foreign body injury) and the normal duodenal wall concealed the toothpick. This fact reminds us of that it is possible to miss an object when contrast-enhanced CT images are used alone, and renewed our appreciation of the value of unenhanced CT. The sensitivity of CT in detecting ingested 
toothpicks is only $42.6 \%$ (1). A careful interpretation of unenhanced CT images may contribute to improving the detection of accidentally-swallowed toothpicks.

One should keep in mind the fact that a wooden foreign body without water absorption appears different. CT shows a dried wooden object as a low density structure due to the influence of air (8). When the time interval between the ingestion of a toothpick and the CT examination is short, the object is likely to appear as a linear low density structure. Matsubara et al. reported a case of an accidentallyswallowed toothpick in which a CT scan was performed the day after ingestion, and the toothpick appeared as a distinct low density linear structure (11). In such situations, wide window settings can facilitate the depiction of an air-filled toothpick and reduce the possibility of overlooking the object.

We have reported a case of small bowel obstruction due to the accidental ingestion of a toothpick. The object was identified on CT and was removed endoscopically. Although CT is reported to show low sensitivity in the detection of swallowed toothpicks, a thorough interpretation, especially using unenhanced CT, should play a crucial role in making a diagnosis of toothpick ingestion.

\section{The authors state that they have no Conflict of Interest (COI).}

\section{References}

1. Steinbach C, Stockmann M, Jara M, Bednarsch J, Lock JF. Accidentally ingested toothpicks causing severe gastrointestinal injury: a practical guideline for diagnosis and therapy based on 136 case reports. World J Surg 38: 371-377, 2014.

2. Budnick LD. Toothpick-related injuries in the United States, 1979 through 1982. JAMA 252: 796-797, 1984.

3. Henderson CT, Engel J, Schlesinger P. Foreign body ingestion: review and suggested guidelines for management. Endoscopy 19: 68-71, 1987.

4. Velitchkov NG, Grigorov GI, Losanoff JE, Kjossev KT. Ingested foreign bodies of the gastrointestinal tract: retrospective analysis of 542 cases. World J Surg 20: 1001-1005, 1996.

5. Robert B, Bartoli E, Fumery M, et al. Duodenal perforation due to toothpick perforation, an uncommon cause of chronic abdominal pain. Endoscopy 44 (S02): E27-E28, 2012.

6. Li SF, Ender K. Toothpick injury mimicking renal colic: case report and systematic review. J Emerg Med 23: 35-38, 2002.

7. Chong LW, Sun CK, Wu CC, Sun CK. Successful treatment of liver abscess secondary to foreign body penetration of the alimentary tract: a case report and literature review. World J Gastroenterol 20: 3703-3711, 2014.

8. Hansen JE, Gudeman SK, Holgate RC, Saunders RA. Penetrating intracranial wood wounds: clinical limitations of computerized tomography. J Neurosurg 68: 752-756, 1988.

9. Venkatesh SH, Venkatanarasimha Karaddi NK. CT findings of accidental fish bone ingestion and its complications. Diagn Interv Radiol 22: 156-160, 2016.

10. Goh BKP, Tan YM, Lin SE, et al. CT in the preoperative diagnosis of fish bone perforation of the gastrointestinal tract. AJR Am J Roentgenol 187: 710-714, 2006.

11. Matsubara M, Hirasaki S, Suzuki S. Gastric penetration by an ingested toothpick successfully managed with computed tomography and endoscopy. Intern Med 46: 971-974, 2007.

The Internal Medicine is an Open Access article distributed under the Creative Commons Attribution-NonCommercial-NoDerivatives 4.0 International License. To view the details of this license, please visit (https://creativecommons.org/licenses/ by-nc-nd/4.0/).

(C) 2017 The Japanese Society of Internal Medicine http://www.naika.or.jp/imonline/index.html 